\title{
REDUCTION OF LOCAL ANESTHETIC VOLUME WITH EXPERIENCE IN YEARS IN ULTRASOUND GUIDED INFRACLAVICULAR BRACHIAL PLEXUS BLOCK
}

\author{
YENIOCAK T., CANBOLAT N. \\ Baltalimani Metin Sabanci Bone and Joint Diseases Education and Research Hospital, Istanbul, TURKEY \\ Department of Anaesthesiology
}

\section{Backround and aims:}

Infraclavicular brachial plexus block (ICB) is commonly used in upper extremity surgeries with ultrasonography guidance (USG). In recent years the use of ultrasonography improved the application of peripheral nerve block with easily monitoring the distribution of injected local anesthetic (LA) by detecting nerve localization, visualization of vascular structures and pleural border, visually controlling the needle tip, and dissemination of the injected LA. We aimed to share our experiences of USGICB for upper extremity surgeries and decreasing LA volume as physicians experience increased in years.

\section{Methods:}

With approval from the ethics committee; age, gender, height, weight, operation type, ASA score, LA dose, complications and block success rate of USGICB anesthesia records were examined between November 2011 and March 2015. 40 months divided in 4 groups chronologically. The anesthesiologists who applied the blocks were 9 people who had the same education and had equal experience of USGICB. From the point of inexperienced period LA dose and success rate, failure and complications were recorded. LA mixture was prepared with $\% 1$ lidocaine and $\% 0.25$ bupivacaine.

\begin{tabular}{|c|c|c|c|c|c|c|c|c|c|c|c|c|c|c|}
\hline & & \multicolumn{3}{|c|}{ Group I } & \multicolumn{3}{|c|}{ Group II } & \multicolumn{3}{|c|}{ Group III } & \multicolumn{3}{|c|}{ Group IV } & \multirow{2}{*}{$p$} \\
\hline & & Mean: & d. $/ n-\%$ & Med & Mean: & $d . / n-\%$ & Med & Mean: & s.d. $/ n-\%$ & Med & Mean: & s.d. $/ n-\%$ & Med & \\
\hline \multicolumn{2}{|l|}{$\overline{\text { Age }}$} & \multicolumn{2}{|c|}{$38,2 \pm 14,7$} & \multirow{3}{*}{35} & \multicolumn{2}{|c|}{$38,6 \pm 14,9$} & \multirow[t]{3}{*}{37} & \multicolumn{2}{|c|}{$35,9 \pm 14,7$} & \multirow[t]{3}{*}{33} & \multicolumn{2}{|c|}{$35,7 \pm 14,1$} & 33 & 0,000 \\
\hline \multirow{2}{*}{ Gender } & Female & 227 & $36,1 \%$ & & 239 & $35,6 \%$ & & 215 & $29,9 \%$ & & 243 & $26,0 \%$ & & \multirow{2}{*}{0,000} \\
\hline & Male & 401 & $63,9 \%$ & & 433 & $64,4 \%$ & & 505 & $70,1 \%$ & & 690 & $74,0 \%$ & & \\
\hline \multicolumn{2}{|l|}{ BMI } & \multicolumn{2}{|c|}{$26,3 \pm 4,8$} & 26 & \multicolumn{2}{|c|}{$26,1 \pm 4,9$} & 26 & \multicolumn{2}{|c|}{$26,2 \pm 5,1$} & 25 & \multicolumn{2}{|c|}{$25,9 \pm 4,6$} & 25 & 0,417 \\
\hline \multirow{4}{*}{ ASA } & I & 341 & $54,3 \%$ & & 331 & $49,3 \%$ & & 420 & $58,3 \%$ & & 633 & $67,8 \%$ & & \multirow{4}{*}{0,041} \\
\hline & ॥ & 263 & $41,9 \%$ & & 324 & $48,2 \%$ & & 285 & $39,6 \%$ & & 285 & $30,5 \%$ & & \\
\hline & III & 22 & $3,5 \%$ & & 17 & $2,5 \%$ & & 14 & $1,9 \%$ & & 14 & $1,5 \%$ & & \\
\hline & IV & 2 & $0,3 \%$ & & 0 & $0,0 \%$ & & 1 & $0,1 \%$ & & 1 & $0,1 \%$ & & \\
\hline
\end{tabular}

\section{Results:}

2953 patients underwent USGICB. In first 10 months process 628 , second 10 months 671 , third 10 months 719 and last 10 months 932 cases were detected. The patient demographic charecteristics (age, BMI) ASA classifications are shown in Table I. There were no significant differences between the groups. Although the LA volume applied as the physician experience increased over the years was not statistically significant, A reduction of volume use over $30 \mathrm{ml}$ was observed in Groups II (32.7 $\pm 4.1 \mathrm{~mL})$, III $(32.4 \pm 3.8 \mathrm{~mL})$ and IV $(32.0 \pm 4.0 \mathrm{~mL})$ compared to the Group I $(33.7 \pm 4.2 \mathrm{~mL})$. (Table II) In group I (3.2\%), the failure rate was higher than group $\mathrm{II}(1.2 \%)-\mathrm{III}(0.7 \%)$ and group IV $(0.5 \%)(p<0.05)$. The failure rates of Group II-III-IV did not differ significantly.(Table III)
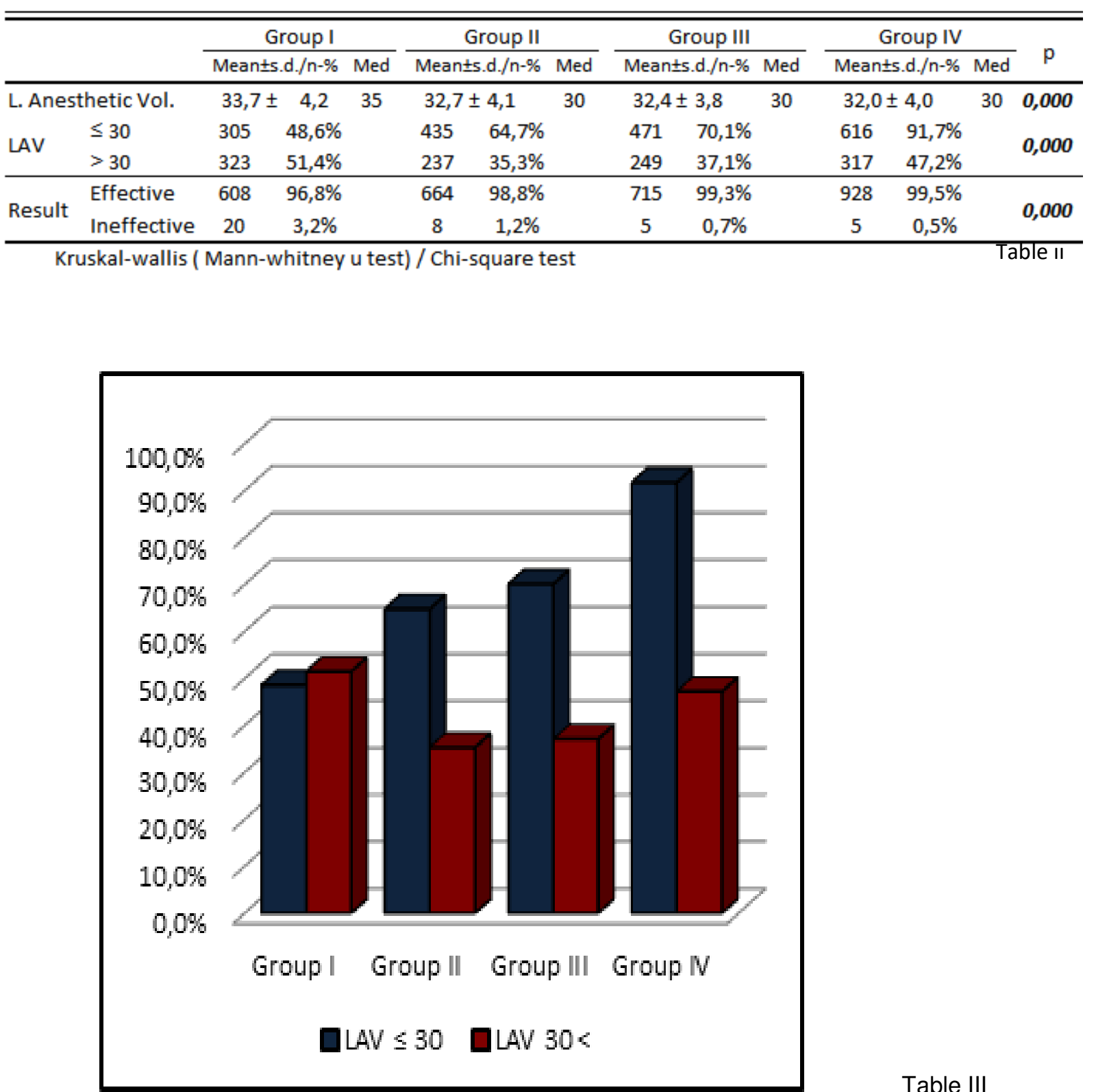

Table III

\section{Conclusions:}

Since our study was retrospective; VAS scoring, postoperative onset time of analgesic requirement, motor block duration were not assessed. Adding these parameters can help to find more comprehensive findings. 